\title{
Klinische Symptomatik von Schlaganfällen
}

\author{
Peter Marx \\ Berlin \\ psychoneuro 2005; 31 (5): 249-255
}

$\mathrm{M}$ akroangiopathische Insulte entstehen durch Verschluss einer hirnversorgenden $\mathrm{Ar}$ terie in ihrem extrazerebralen Verlauf. Häufigste Ursache ist eine Embolie, die entweder aus dem Herzen oder von arteriosklerotisch veränderten Gefäßen (arterio-arterielle Embolie) stammt. Autochthone thrombotische Verschlüsse sind selten. Das Resultat ist der Territorial- oder Astverschlussinfarkt, seltener der Grenzgebietsinfarkte zwischen Anterior, Media oder Posterior. Mikroangiopathische Insulte haben höchst selten eine embolische Genese. Sie entstehen durch Verschluss intraparenchymaler kleiner Arterien und Arteriolen durch Lipohyalinose oder Arteriolosklerose. Das Resultat ist der lakunäre Infarkt ( $<1,5 \mathrm{~cm}$ Durchmesser) oder die diffuse Marklagerangiopathie.

\section{Verlaufsformen ischämischer Insulte}

Klinisch können, gemessen an der Dauer der Symptomatik, unterschiedliche Verlaufsformen unterschieden werden.

- Passagerer ischämischer Insult (TIA, transient ischemic attack)

- Reversibler ischämischer Insult (RIND, reversible ischemic neurological deficit)

- Progredienter ischämischer Insult

Unter dem Begriff Schlaganfall werden vier unterschiedliche zerebrovaskuläre Krankheiten zusammengefasst: ischämischer Insult, Hirnblutung, Subarachnoidalblutung, Hirnvenenthrombose. Gemeinsames Charakteristikum der klinischen Symptomatik dieser Erkrankungen ist der meist schlagartige, apoplektiforme Beginn. Neurologische Symptome entsprechen dem von der jeweiligen Erkrankung erfassten Hirnteil und lassen keine sicheren Rückschlüsse auf die Ätiologie der Störung zu. So ist z.B. ein ischämischer Insult allein durch Anamnese und klinische Symptomatik nicht von einer Hirnblutung zu differenzieren.

- Vollendeter ischämischer Insult - mit minimalem Defizit (Minimal Stroke)

- mit schwerem Defizit.

TIA umschreibt ischämisch bedingte neurologische Ausfälle, die sich innerhalb von 24 Stunden vollständig zurückbilden. Die Wahl der Zeitspanne ist unglücklich, da sich die meisten TIA innerhalb von ca. 15 Minuten zurückbilden und dann selten einen Hirninfarkt hinterlassen. Hält die Symptomatik länger an, findet man umso häufiger Hirninfarkte im MRT, je länger Ausfälle bestanden.

Die Diagnose reversibler ischämischer Insult (Rückbildung der Symptome nach 24 Stunden) sollte verlassen werden, da es sich fast immer um einen Minimal Stroke handelt. Eine progrediente Symptomatik ist für ischämische Insulte ungewöhnlich und muss immer an andere Ursachen denken lassen. Vollendeter ischämischer Insult be- zeichnet den Hirninfarkt. Aus klinischen und prognostischen Gesichtspunkten werden TIA und Minimal Stroke oft zusammengefasst.

\section{Klinische Symptomatik mikroangiopathischer Insulte}

Mikroangiopathien können lange asymptomatisch oder unbemerkt bleiben. Dies trifft insbesondere für die Leukoaraiose $z u$.

\section{Lakunäre Syndrome}

Klinisch sind sehr viele lakunäre Syndrome beschrieben worden (4). Sie sind ätiologisch unspezifisch und kommen auch bei anderen Infarkttypen, bei kleinen Blutungen und sogar bei Tumoren vor. Mit der seltenen Ausnahme bei Lakunen im Thalamus fehlen neuropsychologische Symptome. Die wichtigsten, weil häufigsten, klinischen Syndrome sind:

- Rein motorischer Schlaganfall (pure motor stroke) 
- Rein sensibler Schlaganfall (pure sensory stroke)

- Senso-motorischer Schlaganfall (sensorimotor stroke)

- Ataktische Hemiparese (ataxic hemiparesis)

- Reine Dysarthrie

- Dysarthrie mit Handungeschicklichkeit (dysarthria-clumsyhand-syndrome)

- Hemichorea, Hemiballismus.

Sehr selten wurden auch einseitige Dystonien, Asterixis und Tremor beschrieben.

Die rein motorische Hemiparese betrifft mindestens zwei der drei Körperregionen (Gesicht, Arm, Bein), wobei das Gesicht am häufigsten ausgespart ist. Eine rein motorische Monoparese ist dagegen fast immer kortikalen Ursprungs. Die rein sensible Hemisymptomatik ist charakteristisch für Thalamuslakunen. Die Patienten geben Taubheits-, Hitze- oder Schweregefühl oder das Gefühl von „Ameisenlaufen“ einer Körperseite an. Eine sensomotorische Hemiparese sollte nur diagnostiziert werden, wenn sorgfältig kortikale Syndrome ausgeschlossen worden sind. Bei der ataktischen Hemiparese findet man kontralateral zur Läsion pyramidale und zerebelläre Störungen. Die Lakune ist meist im Brückenfuß, seltener in der Corona radiata, in den Stammganglien oder im Thalamus lokalisiert. Das Dysarthria-clumsyhand-Syndrom wird meist von einer leichten fazialen Parese begleitet. Die Läsion liegt fast immer im vorderen Anteil der inneren Kapsel.

Subkortikale arteriosklerotische Enzephalopahtie (M. Binswanger): Leukoaraiose und multiple Lakunen sind die morphologischen Grundlagen des M. Binswanger. Klinisch findet man meist unscharfe neurologische Ausfälle und eine Demenz. Im CCT sieht man neben Lakunen periventrikuläre Hypodensitäten, die aber auch bei anderen Erkrankungen, wie z. B. CADASIL, Leukodystrophien etc. vorkommen.

\section{ב.}

Klinische Symptomatik makroangiopathischer Insulte Makroangiopathische oder kardioembolische Infarkte sind immer zu vermuten, wenn neben Ausfällen von Seiten der langen Bahnen auch neuropsychologische Symptome oder eine homonyme Hemianopsie vorliegen $(2,6)$.

\section{Karotisstromgebiet}

Infarkte bei Interna-Verschlüssen oder Stenosen betreffen selten das gesamte Versorgungsgebiet. Häufiger sind umschriebene Insulte im Versorgungsgebiet ihrer Äste.

\section{A. ophthalmica}

Amaurosis fugax umschreibt eine meist nur Sekunden bis Minuten anhaltende monokuläre Sehstörung. Der Patient bemerkt eine graue Unschärfe oder einen völligen Sehverlust auf einem Auge oder in Teilen des Gesichtsfeldes eines Auges, oft geschildert „als wenn eine Klappe von oben oder unten zufällt". Positivphänomene, wie Phosphene, Fortifikationsmuster etc. sind für eine Amaurosis fugax ungewöhnlich. Bleibende Gesichtsfeldausfälle oder völlige Erblindung entstehen durch Netzhautinfarkte oder ischämische Läsion des Sehnerven (anteriore oder posteriore ischämische Optikusneuropathie). Seltene Symptome sind die passagere Erblindung bei hellem Licht (pathologische PhotostressReaktion) und die Rubeosis iridis mit Neovaskularisation der Iris, Sekundärglaukom, Pupillenerweiterung und proliferativer Retinopathie und konsekutiver Erblindung.

\section{A. chorioidea anterior}

Verschlüsse dieser Arterie können asymptomatisch verlaufen, $\mathrm{zu}$ rein motorischer, rein sensorischer oder ataktischer Hemiparese mit sensiblen Ausfällen oder zu kontralateraler sensomotorischer Hemiparese mit homonymer Hemi- oder Quadrantenanopsie zur Gegenseite führen. Das Bild gleicht dann einem Mediainfarkt und kann auch von neuropsychologischen Störungen (visueller Hemineglect, konstruktive Apraxie und Sprachstörungen) begleitet sein.

\section{A. cerebri anterior}

Bei Anterior-Totalinfarkten (einschl. der Heubner'schen Arterie) findet man kontralateral eine sensomotorische Hemiparese. Die Sensi- bilitätsstörung betrifft besonders propriozeptive Funktionen. Sind Gyrus cinguli und Fornix links- oder beidseitig betroffen, sind Gedächtnisverlust und Korsakow-Syndrom weitere Folgen. Die häufigeren Verschlüsse distal der A. comm. ant. führen zu Infarkten der Mantelkantenregion mit kontralateraler beinbetonter, sensomotorischer Hemiparese, Urininkontinenz, kontralateralem pathologischem Greifreflex, der auch den Fuß erfassen kann, der dann „wie festgesaugt“ am Boden klebt, Saugreflex und Gegenhalten. Bei Beteiligung des Balkens und seiner Projektionen findet man vielfältige neuropsychologische Syndrome: Alien-Hand-Syndrom, linksseitige ideomotorische Apraxie und Dysgraphie, taktile Anomie links etc. Linksseitige Anteriorinfarkte führen zu Reduktion der Spontansprache, Wortfindungsstörungen, transkortikaler Aphasie, Dysarthrie und homolateraler Apraxie, rechtshirnige zu linksseitigem motorischem Neglect, Abulie, Apathie und Verwirrtheit. Außerdem sind bei Verschlüssen der proximal abgehenden Äste für das Frontalhirn Agitiertheit, Angst und Schlaflosigkeit beschrieben worden.

Ausgedehnte bilaterale Anteriorinfarkte verursachen initial Bewusstseinsstörungen bis zum Koma, die in einen akinetischen Mutismus und später in schwere Antriebsstörungen übergehen können. Zusätzlich finden sich eine Paraparese und langanhaltende Inkontinenz.

\section{A. cerebri media}

Der Mediatotalinfarkt bewirkt eine kontralaterale, durchgehende, d.h. Gesicht, Arm und Bein betreffende, sensomotorische Hemiparese und eine homonyme Hemianopsie zur Gegenseite. Eine initiale Blicklähmung zur Gegenseite mit tonischer Deviation von Kopf und Augen zur gesunden Seite (der Kranke blickt - im Gegensatz zur pontinen Blicklähmung - zur nicht gelähmten Seite bzw. zum Herd) beruht auf einem Tonusüberwiegen der gesunden Hemisphäre und signalisiert eine schlechte Prognose.

Infarkte der sprachdominanten Hemisphäre führen initial zu globa- 


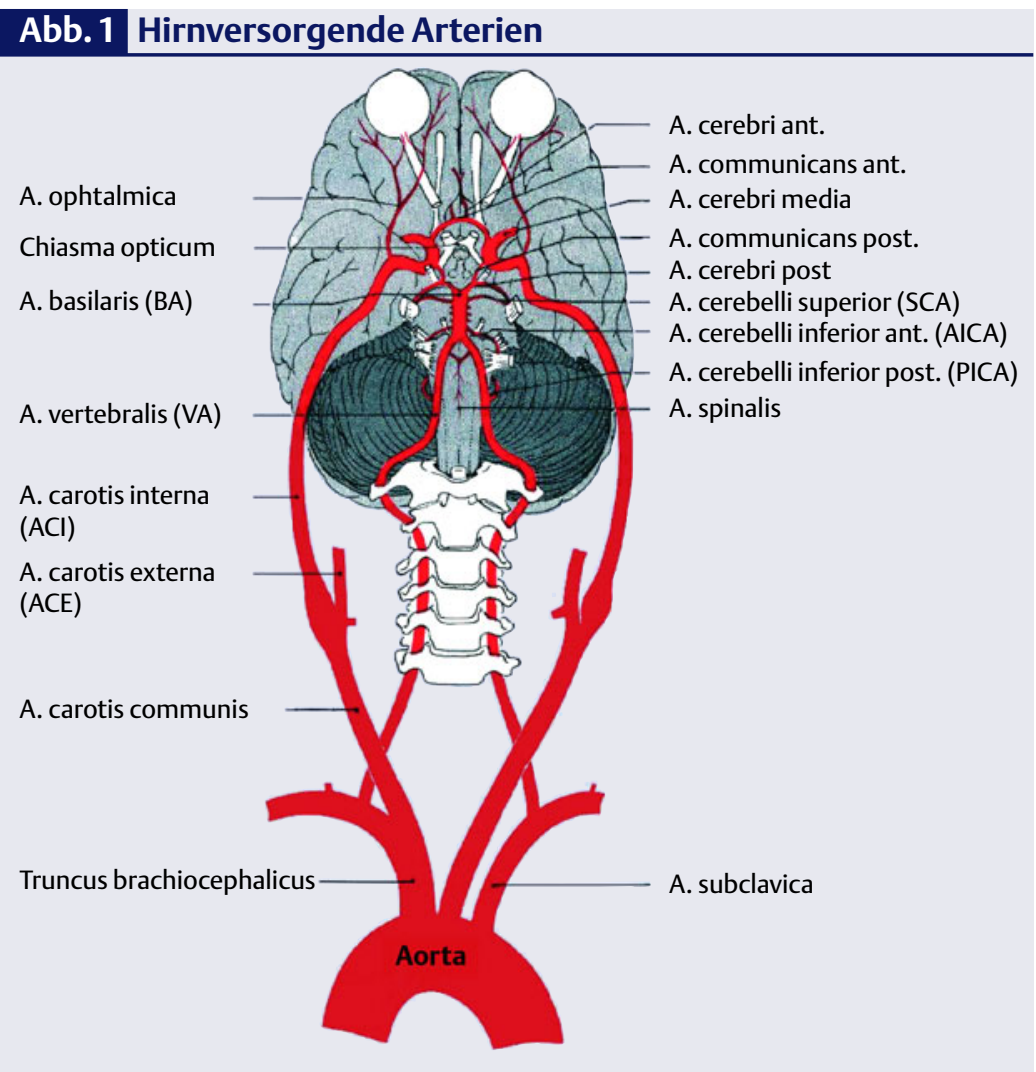

ler Aphasie, oftmals mit Rückbildung zu Broca-Aphasie. Auch das gestische Kommunikationsverständnis ist anfangs oft aufgehoben. Infarkte der nicht sprachdominanten Hemisphäre verursachen kontralateralen, multimodalen Neglect, der sich meist zurückbildet. Leichtere Aufmerksamkeitsstörungen, wie z.B. ein visuelles Hemiextinkt, können jedoch persistieren und z. B. Fahrtüchtigkeit ausschließen. Leichte Bewusstseins- und Vigilanzstörungen kommen bei Insultbeginn häufig vor und beruhen wahrscheinlich auf Diaschisis, d.h. Nichtaktivierung frontaler und kontralateraler Strukturen. Bewusstseinsstörungen nach zwei bis vier Tagen deuten auf Hirnschwellung (sog. maligner MediaInfarkt), massive Einblutung oder extrazerebrale Ursachen hin.

Mediateilinfarkte: Verlegt der Verschluss nur den Abgang der lenticulostriären Arterien kann es bei guter kortikaler Kollateralisation zum Bild des großen (nicht lakunären) Stammganglieninfarkts (Basalganglien- oder auch Linsenkerninfarkt) kommen. Klinisch findet man eine sensomotorische kontrala- terale Hemiparese mit oder ohne homonyme Hemianopsie.

Mediaastverschlüsse distal des Abgangs der Stammganglienarterien rufen unterschiedliche Ausfallsmuster hervor.

Sind die vorderen Mediaäste betroffen, findet man das typische Bild einer brachio-fazial betonten Hemiparese mit oder ohne Sensibilitätsstörung, die bei Infarkten der sprachdominanten Hemisphäre meist von einer motorischen Aphasie und bucco-fazialen Apraxie begleitet ist. Sensibilitätsstörungen betreffen besonders diskriminative Funktionen.

Bei rechtshemisphärischen Infarkten kommen ein sich meist innerhalb von Tagen zurückbildender Neglect bis zu Anosognosie bei Beteiligung frontaler Strukturen vor. Oft findet man auch vorübergehende Dysprosodie und Dysarthrie.

Bei Verschlüssen der hinteren Mediaäste fehlen oft motorische und sensible Ausfälle ebenso wie Kopf- und Augendeviation. Charakteristisch sind homonyme Hemioder Quadrantenanopsie und bei linkshirnigen Infarkten Wernicke-
Aphasie, bei rechtshirnigen multimodaler Neglect und gelegentlich Amusie.

Bei distalen Mediaastverschlüssen findet man eng umschriebene Ausfälle, wie isolierte faziale Parese, Monoparese des kontralateralen Armes und gelegentlich eine ulnar betonte Monoparese der kontralateralen Hand sowie isolierte neuropsychologische Syndrome.

Vertebro-basiläres Stromgebiet

Die Ursachen ischämischer Läsionen im hinteren Kreislauf entsprechen denen im Karotisstromgebiet. Die klinische Symptomatik entspricht den von der Ischämie betroffenen Strukturen des Hirnstammes, des Thalamus und den von der Posterior versorgten Großhirnarealen. Der Begriff „vetebrobasiläre Insuffizienz“, bezeichnet eine diffuse Mangeldurchblutung und sollte aufgegeben werden

Bei passageren Insulten im hinteren Stromgebiet ist eine genaue lokalisatorische Zuordnung schwierig. Geklagt werden Schwindel, Doppelbilder, motorische Störungen (oft bilateral), Ataxie, dissoziierte Sensibilitätsstörungen und homonyme Gesichtsfeldausfälle.

Schwindel allein reicht nicht aus, eine Ischämie im vertebrobasilären Stromgebiet $\mathrm{zu}$ diagnostizieren. Wird der Schwindel aber von einem oder mehreren der genannten Symptome begleitet oder gefolgt, ist dies ein deutlicher Hinweis auf eine Durchblutungsstörung. Dies gilt besonders dann, wenn sich in unmittelbarem Anschluss an Hirnstammsymptome eine homonyme oder auch bilaterale Gesichtsfeldeinschränkung einstellt (typisch für einen durch die Basilaris in die Posterior wandernden Embolus).

\section{Hirnstamm- und Kleinhirn- infarkte}

Verschlüsse im Bereich der Vertebralarterien, der Basilaris und ihrer Äste führen zu außerordentlich vielfältigen Symptomkonstellationen $(1,6)$, aus denen viele mit Eigennamen belegte Syndrome konstituiert wurden. Logischer ist eine Einteilung nach den Gefäßversorgungsgebieten. 
$A$. vertebralis und $A$. cerebelli inferior posterior (PICA)

Infarkte betreffen vor allem die dorsolateralen Versorgungsgebiete der Medulla oblongata und das Kleinhirn (Wallenberg-Syndrom). Zusätzlich kann eine vertikale Achsendivergenz der Bulbi bei ansonsten normaler Augenbeweglichkeit (skew deviation) vorkommen. Eine kontralaterale Pyramidenbahnstörung weist auf einen begleitenden Verschluss eines basalen Perforatorgefäßes hin.

Kleinhirninfarkte bei PICA-Verschluss mit oder ohne Hirnstammbeteiligung können im Rahmen des sich entwickelnden Ödems zu intrakranieller Drucksteigerung mit tödlicher Kompression des Hirnstamms führen (sog. maligner Kleinhirninfarkt).

\section{A. cerebelli inferior anterior (AICA)}

Infarkte betreffen den dorsolateralen Pons und weisen zusätzlich zu den Symptomen des WallenbergSyndroms eine homolaterale faziale Parese und Ertaubung auf.

\section{A. cerebelli superior (SCA)}

Infarkte führen zu Nystagmus zur Herdseite, homolateraler Extremitätenataxie, Gangataxie, Tremor und Horner-Syndrom. Kontralateral findet sich eine Störung der Temperatur- und Schmerzempfindung.

\section{A. basilaris}

Verschluss kurzer perforierender Äste im Brückenbereich (Rami ad pontem) führt zu lakunaren Syndromen, wie Dysarthria Clumsy HandSyndrome oder rein motorischer oder ataktischer Hemiparese. Sind lange mediale Äste betroffen, kann eine internukleäre Ophthalmoplegie durch Ausfall des medialen Längsbündels resultieren.

Verschlüsse der kurzen zirkumferierenden Arterien verursachen paramediane Infarkte $z$. B. mit pontiner Blicklähmung. Ist die Pyramidenbahn mitbetroffen, blickt der Kranke im Gegensatz zur Blicklähmung bei Großhirninfarkten zur gelähmten Seite, d.h. in Richtung auf den Herd. Noch seltener ist das Eineinhalb-Syndrom mit Blicklähmung zur betroffenen Seite und ipsilateraler internucleärer Ophthalmoplegie.

Bei Basilaris-Verschlüssen besteht immer akute Lebensgefahr

Verschlüsse im Bereich der Vereinigungsstelle der Vertebralarterien (Kaudale Basilaristhrombose) führen zu Ausfällen der Pyramidenbahn, der sensiblen Trakte, der kaudalen Hirnnerven und zu ataktischen Störungen durch Läsion des Kleinhirns oder seiner Bahnen im Hirnstamm. Die resultierenden Symptome sind meist bilateral, können aber auch unterschiedliche Schwerpunkte auf einer Seite haben. Das Bewusstsein ist erhalten und nur bei Hirndrucksymptomatik gestört.

Die Basilaristhrombose im mittleren Abschnitt führt zu bilateralen pontinen Ausfällen mit Tetraparese, blickmotorischen Störungen und sensiblen Ausfällen (Locked-In-Syndrom). Der Patient ist wach, kann aber wegen einer Tetraplegie und der Unmöglichkeit, seine kaudalen Hirnnerven $\mathrm{zu}$ innervieren, ausschließlich durch vertikale Blickbewegungen Kontakt mit der Umwelt aufnehmen. Bei kurzstreckigem Verschluss und gutem kollateralen $\mathrm{Zu}$ fluss aus dem vorderen Kreislauf können die klinischen Ausfälle aber auch erstaunlich gering sein. Nach erfolgreicher Thrombolyse ist die Rezidivgefahr hoch.

Verschlüsse der Basilarisspitze sind meist embolischer Genese und führen zum Syndrom der Basilarisspitze. Da die vigilanzsteuernden Systeme im oberen Hirnstamm und/oder ihre Umschaltstelle im medialen Thalamus von der Ischämie betroffen sind, haben die Patienten meist Bewusstseinsstörungen (initial Bewusstseinsverlust oder -trübung oder auch Erregungszustände). Oft bleibt ein vermehrtes Schlafbedürfnis für lange Zeit bestehen. Charakteristisch sind Störungen der vertikalen Augenbewegungen, die durch die Dissoziation eines in horizontaler Ebene erhaltenen, in vertikaler Ebene erloschenen okulozephalen Reflexes (Puppenkopfphänomen) auch beim Bewusstlosen erkannt werden können. Weiterhin bestehen unterschiedliche Pupillen- innervationsstörungen. Paresen sind selten und meist reversibel. Einschluss des N. subthalamicus kann zu Hemiballismus führen. Optische Halluzinationen können vorkommen.

\section{Posterior Infarkte}

Verschlüsse im P1-Abschnitt können zu Infarkten im Mittelhirnfuß, medialen Anteilen des Mittelhirnes und Thalamus mit kontralateraler sensomotorischer Hemiparese, komplexen Augenmotilitätsstörungen besonders in der vertikalen Ebene und Bewusstseinsstörungen führen. Mittelhirnfußinfarkte hinterlassen gelegentlich ein WeberSyndrom (homolateral Okulomotoriusparese, kontralateral Hemiparese).

Sehr viel häufiger sind embolische Verschlüsse der weiter distalen Gefäßabschnitte, die zu Okzipitallappeninfarkten mit homonymer Hemioder Quadrantenanopsie führen. Einfache visuelle Halluzinationen (Photopsien oder Phosphene) kommen bei kleinen Infarkten im Kortex des Interhemisphärenspaltes vor und indizieren eine gute Restitutionsprognose des Gesichtsfeldausfalls (3). Etwa 10\% der Patienten berichten allerdings meist nur auf gezieltes Befragen - komplexe visuelle Halluzinationen oder Pseudohalluzinationen (Personen, Gesichter, Tiere, Gegenstände etc., gelegentlich auch Verdoppelungen, Nachbilder und Palinopsie) im hemianopen Gesichtsfeld.

Bei linkshirnigen Posteriorinfarkten können außerdem Farbagnosie, Alexie ohne Agraphie oder Wortfindungsstörungen gefunden werden, während rechtsseitige Infarkte visuelle Orientierungs- und Gedächtnisstörungen zur Folge haben. Sind auch die Äste zum medialen Temporallappen und damit Hippocampus und Fornix betroffen, kommt es zu Merkfähigkeits- und Orientierungsstörungen.

Bilaterale Infarkte führen zu ausgedehnten beidseitigen Gesichtsfeldausfällen bis zur völligen Erblindung bei erhaltenen Pupillenreflexen (sog. Seelenblindheit) und Anosognosie (Anton-Syndrom). Prosopagnosie und Simultanagnosie sind selten. 


\section{- Hirnblutungen}

Hirnblutungen entstehen durch Riss einer intraparenchymatösen Arterie und dürfen nicht mit sekundär hämorrhagisch umgewandelten Hirninfarkten verwechselt werden. Ihre neurologische Ausfallssymptomatik entspricht der Lokalisation und dem Ausmaß der Blutung bzw. dem durch lokalen Druck oder Zerreißung betroffenen Hirnareal. Kopfschmerzen fehlen bei rein intraparenchymatösen Blutungen und entstehen erst durch Einbruch der Blutung in den Liquorraum oder bei intrakranieller Drucksteigerung. Ischämische Insulte und Hirnblutungen sind daher klinisch nicht sicher zu unterscheiden. Epileptische Anfälle sind in der Frühphase von Lobärblutungen häufiger als bei Infarkten.

Basalganglienblutungen (Striatumblutungen) beginnen mit Übelkeit, Erbrechen, zunehmender Hemiparese, Blickwendung zur betroffenen Hemisphäre (=Blicklähmung nach kontralateral) und oft auch globaler Aphasie bei linkshirnigen Blutungen. Kleine Blutungen können dagegen wie ein TIA oder leichter ischämischer Insult verlaufen. Große Blutungen brechen oft in den Ventrikel ein oder wühlen sich in die Hemisphäre vor. Thalamus- und Brückenblutungen rufen die lokalisationsspezifischen Ausfallsmuster hervor. Kleinhirnblutungen sind wegen ihres raumfordernden Charakters in der hinteren Schädelgrube immer lebensbedrohlich.

Eine Hirndrucksymptomatik beginnt mit Kopfschmerz, Meningismus, Erbrechen und zunehmender Bewusstseinstrübung. Später stellen sich bei supratentoriellen Hämatomen die Symptome der oberen Einklemmung (einseitige Mydriasis, Beuge-Synergien, Beuge-Strecksynergien, Bewusstseinsverlust, vegetative Dysregulationen) ein. Druckerhöhung in der hinteren Schädelgrube führt zu Verlust des Puppenkopfphänomens, Erlöschen der okulomotorischen Reaktion auf Kaltspülung des Ohres, Verlust des Kornealreflexes und schließlich zu bulbärer Symptomatik, Zusammenbruch der Atmungs- und Kreislaufregulation, Tonusverlust der Muskulatur, Poikilothermie und schließlich dissozi- iertem Hirntod. Hämatome der hinteren Schädelgrube führen oft ohne die Warnzeichen der oberen Einklemmung zur Kompression der Medulla oblongata im Foramen occ. magnum.

\section{Subarachnoidalblutungen (SAB)}

Vorboten, wie plötzliche Kopfschmerzen oder Augenmuskelparesen in Folge Aneurysmaausdehnung oder Sickerblutung sind selten. Üblicherweise kommt es plötzlich zu einem äußerst schweren (noch nie erlebten) Kopfschmerz, der sich innerhalb von Sekunden oder ein bis zwei Minuten von Nacken oder Stirn auf den ganzen Kopf ausbreitet und nach Stunden auch im Rücken (meningeale Reizung) verspürt wird. Ein initialer Bewusstseinsverlust kommt bei etwa der Hälfte der Patienten vor. Neurologische Ausfälle sind nicht obligat, vegetative Dysregulationen (Übelkeit, Erbrechen, Kreislaufdysregulation) sind häufig. Meist findet man einen Meningismus (sein Fehlen schließt die SAB aber nicht aus!), im Augenhintergrund lassen sich gelegentlich frische, papillennahe Blutungen aufzeigen. Mydriasis oder Okulomotoriuslähmung weist auf ein supraclinoidales Aneurysma der Karotis int. am Abgang der A. comm. post hin. Neurologische Fokalsymptome, epileptische Anfälle, Verwirrtheitsoder Erregungszustände sind in der Frühphase selten, können sich aber ebenso wie neurologische Herdsymptome und sekundäre Bewusstseinsstörungen im Rahmen der SABtypischen Komplikationen (Rezidivblutungen, Hydrozephalus malresorptivus und Vasospasmen) entwickeln. Ein Drittel der Patienten entwickelt eine inadäquate ADH-Ausschüttung, bis zu 80\% EKG-Veränderungen und andere kardiale Probleme.

\section{- Hirnvenenthrombosen}

Zerebrale venöse Thrombosen gehören zu den wahrscheinlich am häufigsten übersehenen Hirndurchblutungsstörungen, von denen sie etwa $1 \%$ ausmachen. Man unterscheidet septische von aseptischen Thrombosen.

\section{Aseptische Hirnvenen- und} Sinusthrombosen

Die klinische Symptomatik ist sehr vielgestaltig (5). Sie setzt oft nicht plötzlich ein, sondern entwickelt sich langsam progredient oder schubweise. Es gibt jedoch auch abruptes Einsetzen von Symptomen. Das häufigste und meist auch erste Symptom ist Kopfschmerz. Er entwickelt sich meist subakut, kann aber auch wie bei einer Subarachnoidalblutung perakut einsetzen und wird meist als stark angegeben. Der Schmerz wird oft von Übelkeit, Erbrechen und Sehstörung begleitet. Einfache motorische Anfälle, oft mit postparoxysmaler Lähmung, komplex-fokale Anfälle oder auch generalisierte Krampfanfälle finden sich bei 50\% der Patienten. Fokalsymptome entwickeln sich häufiger als bei arteriellen Durchblutungsstörungen progredient, z.B. Beginn mit einer Monoparese, die sich zu einer Hemiparese ausweitet, der sich neuropsychologische Symptome hinzugesellen. Vigilanzstörungen, psychotische Symptome und Verwirrtheitszustände sind führende Symptome der Thrombosen der inneren Hirnvenen mit Abflussbehinderung aus dem oberem Hirnstamm, dem Thalamus und den mediotemporalen Einzugsgebieten. Sinusthrombosen sind eine häufige Ursache einer Stauungspapille bzw. eines Pseudotumors cerebri.

Septische Sinusthrombosen entstehen meist als Komplikation entzündlicher Prozesse im HNOoder Gesichtsbereich und betreffen die jeweiligen Drainagesinus.

Das Krankheitsbild ist neben Kopfschmerz und den lokalen Symptomen durch allgemeine entzündliche Krankheitssymptome bis hin zur Sepsis gekennzeichnet.

Am häufigsten sind S. transversus und S. sigmoideus in Folge einer Otitis media oder Mastoiditis betroffen. Entzündliche Prozesse in Gesicht (Furunkel, entzündete Insektenstiche etc.), Orbita oder Stirnhöhle können wegen der extra-/intrakraniellen venösen Anastomosen in der Orbita zur Thrombose des S. cavernosus führen. Klinisch finden sich neben Chemosis, Portrusio bulbi und Stauung der Netzhaut- 
venen eine schmerzhafte Ophthalmoplegie und Ausfälle des 1. Trigeminusastes. Der Prozess beginnt oft einseitig, greift dann aber über die Ss. intercavernosi auf die Gegenseite über.

\section{Clinical symptomatology of stroke} Stroke summarizes four different cerebrovascular diseases: ischemic stroke, cerebral hemorrhage, subarachnoid hemorrhage, and cerebral venous thrombosis. The common characteristic of the clinical symptomatology of these disorders is the sudden, apoplectic onset. Neurological symptoms correspond mainly to the involved part of the brain and not to their etiology. Therefore an ischemic stroke can not be safely differentiated from a cerebral hemorrhage by case history and clinical symptomatology alone.

\section{Key Words}

stroke - ischemic stroke - cerebral hemorrhage - subarachnoid hemorrhage - cerebral thrombosis

\section{Literatur}

1. Caplan L, (ed.). Posterior Circulation Disease. Cambridge UK, Blackwell Science; 1996

2. Hartmann A. Klinik des akuten ischämischen Insults. In: Hartmann A, Heiss WD (Hrsg.). Der Schlaganfall. Darmstadt: Steinkopf; 2001, 217-324

3. Kölmel HW. Die homonymen Hemianopsien. Berlin, Springer; 1988

4. Marti-Vilalta JL, Arboix A, Mohr JP. Lacunes. In: Mohr J, Choi DW, Grotta JC, Weir B, Wolf PA (ed.). Stroke. Pathophysiology, Diagnosis and Management. Philadelphia, Churchill Livingstone; 2004: 275-99

5. Masuhr F, Mehraein S, Einhaupl K. Cerebral venous and sinus thrombosis. J Neurol 2004; 251(1): 11-23

6. Mohr J, Choi D, Grotta J, Weir B, Wolf P. Stroke. Pathophysiology, Diagnosis and Management. 4 ed. Philadelphia, Churchill Livinstone; 2004 . Section II, 61-300

\section{Korrespondenzadresse}

Prof. Peter Marx

Terrassenstraße 45

14129 Berlin
Georg Thieme Verlag KG

Rüdigerstraße 14

70469 Stuttgart

Telefon (0711) 89 31-0

Telefax (0711) 8931-322

Verantwortliche Schriftleitung:

Markus Gastpar, Essen

Jürgen Fritze, Pulheim

Erweiterte Schriftleitung:

Günther Faust, Mainz

Manfred Fichter, Prien am Chiemsee

Andreas Hufnagel, Essen

Joachim Morgner, Dresden

Götz-Erik Trott, Aschaffenburg

Wissenschaftlicher Beirat:

Thomas Becker, Günzburg; Stefan Bender,

Marsberg; Horst Berzewski, Berlin; Lothar

Blaha, Deggendorf; Hans-Dieter Brenner,

Bern; Pasquale Calabrese, Bochum; Kle-

mens Dieckhöfer, Bonn; Tilman Elliger,

Köln; Volker Faust, Ravensburg; Franz jo-

seph Freisleder, München; Michael Geyer,

Leipzig; Ralf Gold, Göttingen; Iver Hand,

Hamburg; Kurt Heinrich, Düsseldorf; And-

reas Heinz, Berlin; Paul Hoff, Zürich; Wolf-

ganglost Wiesbaden; Georgluckel, Ber-

lin Siegfried Kameier Merzig; Wilfried

lin; Siegfried Kaumeier, Merzig; Wilfried

burg/München; Matthias R. Lemke, Bonn;

burg/München; Matthias R. Lemke, Bonn;

Walter E. Müller, Frankfurt am Main;

mas Müller, Bochum; Dieter Naber,

Helmut Remschmidt, Marburg; Peter Rie-

Helmut Remschmidt, Marburg; Peter Rie-
derer, Würzburg; Eckart Rüther, Göttin-

gen; Jörn Peter Sieb, Stralsund; Wolfgang

Weig, Osnabrück; Wolfgang Werner,

Merzig; Roland Wörz, Bad Schönborn;

Manfred Wolfersdorf, Bayreuth

Bestellungen: Über den Buchhandel oder direkt beim Verlag

Bezugsbedingungen:

Einzelheft $€ 10,50+$ Porto.

Jahresabonnement $€ 75$,-

inkl. MwSt. und Porto.

Für Medizinstudenten

€ 48,- inkl. MwSt. und Porto.

Ausland zuzüglich Versandkosten

(cash with order) $€ 19,80$ (Europa)

bzw. $€ 52,80$ (Airlift).

Der Abonnementpreis umfasst $12 \mathrm{Ka}$ lendermonate (Mindestlaufzeit). Abonnements laufen weiter, wenn nicht 3 Monate vor Jahresende eine Abbestel3 Monate vor Jahresende
lung beim Verlag vorliegt.

Bei Nichtbelieferung im Falle höherer Cewalt, bei Störungen des BetriebsGewalt, bei Story friedens, Arbeitskampf(Streik, Aussperrung) bestehen keine Ansprüche gegen den Verlag.

Verlag und Copyright:

(C) 2005 by Georg Thieme Verlag, Rüdigerstraße 14, 70469 Stuttgart.

Mit dem Abdruck des Beitrages erwirbt der Verlag das alleinige und ausschließliche Recht für die Veröffentlichung in sämtlichen Publikumsmedien sowie Übersetzungen in fremde Sprachen. Nachdruck, fotomechanische Wiedergabe und Speicherung in den Datenverarbeitungsanlagen, auch auszugsweise, nur beitu nach schiftichung des Verlages. Die Abbildungen in den Beiträgen stammen, wenn nicht anders vermerkt, von den jeweiligen Autoren. Für Inhalt, Vorschau und Titelseite werden Abbildungen aus den beschriebenen Arbeiten verwendet, anderenfalls ist die Herkunft ebenfalls im Inhaltsverzeichnis deklariert. Hinweis für Autoren: Zur Veröffentlichung in der Zeitschrift angenommen werden unveröffentlicht Originalarbeiten, Ubersichtsarbeiten un Kasuistiken, die nicht auch gleichzeitig an anderer Stelle zur Veröffentlichung eingereicht sein dürfen. Richtlinien zur Abfassung des Manuskriptes können bei de Redaktion angefordert werden.

Die eingereichten Arbeiten für Editorial, Schwerpunkt/Brennpunkt und Übersicht/Originalarbeit werden einem pee review der Schriftleitung unterzogen. Der Inhalt der übrigen Rubriken und der Supplements liegt in der Verantwortung der Redaktion.
Redaktionsleitung

Günther Buck

Telefon (0711) 89 31-440

Chefredaktion:

Katrin Wolf

Telefon (0711) 8931-179

Katrin.Wolf@thieme.de

Redaktionsassistenz:

Sabine Bischoff

Telefon (0711) 8931-5 51

Telefax (0711) 8931-322

Internet-Adresse:

http://www.psychoneuro.info

Verantwortlich für den Anzeigenteil:

pharmedia, Anzeigen-

und Verlagsservice $\mathrm{GmbH}$

Anzeigenleitung:

Greta Weller

Telefon (0711) 8931-304

Greta.Weller@pharmedia.de

Zur Zeit gilt Anzeigenpreisliste

1.10 .2004

Zeitschriftenvertrieb:

Telefon (0711) 89 31-321

Herstellung:

Werner Schulz

Telefon (0711) 89 31-331

Satz, Grafik, Layout:

Wolfgang Eckl, Werner Schulz, Karl-Heinz Zobel

Druck und Verarbeitung:

W. Kohlhammer Druckerei GmbH + Co. 70329 Stuttgart

Bankverbindung:

Deutsche Bank Stuttgart

Konto-Nr. 1420 017, BLZ 60070070

Landesbank Baden-Württemberg

Konto-Nr. 2055 723, BLZ 60050101

Postgiro Stuttgart

Konto-Nr. 45 000-705, BLZ 60010070

Erscheinungsweise: monatlich

Regularly listed in EMBASE

Hinweis:

Wie jede Wissenschaft ist die Medizin ständigen Entwicklungen unterworfen. Forschung und klinische Erfahrung erweitern unsere Erkenntnis, insbesondere was Behandlung, medikamentöse The rapie sowie Diagnostik (Laborwerte etc.) anbelangt. Soweit in dieser Zeitschrift Dosierungen, Applikationen oder Laborwerte erwähnt werden, darf der Leser zwar darauf vertrauen, dass Autoren, Herausgeber und Verlag große Sorgfalt darauf verwandt haben, dass diese Andarauf verwand hissensstand gaben dem Wissensstand bei Fertigstellung entsprechen. Für Angaben übe Dosierungsanweisungen, Applikations formen und Laborwerte kann vom Ver lag jedoch keine Gewähr übernommen werden. Jeder Benutzer ist angehalten, durch sorgfältige Prüfung der Beipackzettel der verwendeten Präparate und ggf. nach Konsultation eines Spezialisten festzustellen, ob die dort gegebene Empfehlung für Dosierungen oder die Beachtung von Kontraindikationen Beachtung von Kontraindikationen gegenubber der Angabe in dieser Zeitschrift abweicht. Eine solche Prüfung ist besonders wichtig bei selten verwendeten Präparaten oder solchen, die neu au den Markt gebracht worden sind. Jede Dosierung oder Applikation erfolgt auf eigene Gefahr des Benutzers. Laborwerte müssen immer auf Ihre Plausibilität geprüft werden und sind abhängig vom jeweiligen Testgerät bzw. Testkit. Autoren und Verlag appellieren an jeden Benutzer, ihm auffallende Ungenauigkeiten dem Verlag mitzuteilen. 